\title{
Adenosine Deaminase (ADA) Deficiency Due to Deletion of the ADA Gene Promoter and First Exon by Homologous Recombination between Two Alu Elements
}

\author{
M. L. Markert, J. J. Hutton, D. A. Wiginton, J. C. States, and R. E. Kaufman \\ Department of Pediatrics, Division of Immunology, Department of Medicine, and Department of Biochemistry, Duke University \\ Medical Center, Durham, North Carolina 27710; and Children's Hospital Research Foundation and Department of Pediatrics, \\ University of Cincinnati College of Medicine, Cincinnati, Ohio 45229
}

\begin{abstract}
In $15-20 \%$ of children with severe combined immunodeficiency (SCID), the underlying defect is adenosine deaminase (ADA) deficiency. The goal of this study was to determine the precise molecular defect in a patient with ADA-deficient SCID whom we previously have shown to have a total absence of ADA mRNA and a structural alteration of the ADA gene. By detailed Southern analysis, we now have determined that the structural alteration is a deletion of $\sim 3.3 \mathrm{~kb}$, which included exon 1 and the promoter region of the ADA gene. DNA sequence analysis demonstrates that the deletion created a novel, complete Alu repeat by homologous recombination between two existing Alu repeats that flanked the deletion. The 26-bp recombination joint in the Alu sequence includes the 10-bp " $B$ " sequence homologous to the RNA polymerase III promoter. This is the first example of homologous recombination involving the $B$ sequence in Alu repeats. Similar recombination events have been identified involving Alu repeats in which the recombination joint was located between the $A$ and $B$ sequences of the polymerase III split promoter. The nonrandom location of these events suggests that these segments may be hot spots for recombination.
\end{abstract}

\section{Introduction}

Severe combined immunodeficiency (SCID) ${ }^{1}$ is a syndrome characterized by a profound deficiency in $B$ and $T$ cell function (1). Infants born with this disorder die in the first few years of life unless treated successfully by bone marrow transplantation or kept in a sterile isolation facility $(1,2)$. In most cases of SCID, the underlying molecular defect is unknown. In $15 \%$ of the patients, however, a deficiency of the enzyme adenosine deaminase (ADA, EC 3.5.4.4) is found (2-3) and is thought to be responsible for their immunodeficiency (4).

In recent years the normal ADA cDNA (5-7) and normal ADA gene (8) have been characterized, facilitating studies of the molecular defects in patients with ADA deficiency. The

Address reprint requests to Dr. M. Louise Markert, Box 3068, Duke University Medical Center, Durham, NC 27710.

Received for publication 12 August 1987 and in revised form 25 November 1987.

1. Abbreviations used in this paper: $\mathrm{ADA}$, adenosine deaminase; SCID, severe combined immunodeficiency.

J. Clin. Invest.

(c) The American Society for Clinical Investigation, Inc.

$0021-9738 / 88 / 05 / 1323 / 05 \$ 2.00$

Volume 81, May 1988, 1323-1327 specific mutations found to date have been similar to those found in other enzyme systems. In most patients, Northern RNA analyses have revealed normal-sized ADA mRNA (9), suggesting that most ADA mutant alleles carry a point mutation leading to an inactive or unstable ADA protein. Indeed, in the two patient ADA cDNAs sequenced to date, point mutations affecting amino acid sequence have been found (10-11). In S1 nuclease studies, some patients have been found with abnormal-sized protected fragments, suggesting splicing defects, insertions, or deletions (9).

We previously reported an apparent deletion mutation in a patient with ADA deficiency and SCID who had a major structural alteration in the $5^{\prime}$ end of the ADA gene (12). This patient, R.P., who has no ADA enzyme activity in his lymphocytes, was shown to have no detectable ADA mRNA by Northern RNA analysis, and to have a deletion in the region of the first exon of the ADA gene by Southern DNA analysis (12). The research described in this paper led to the precise definition of the boundaries of the deletion in this patient and the mechanism of the defect, namely, homologous recombination between two repetitive DNA sequences of the Alu family, resulting in the deletion of the ADA promoter and first exon region.

\section{Methods}

Cell lines. B lymphoblastoid cell lines were established from FicollHypaque purified peripheral mononuclear cells by EBV transformation (12-13). The patient, R.P., his mother, and father have been extensively studied clinically at Duke University Medical Center (reference 2, patient 13; reference 14). Enzymatic studies have confirmed the patient's absolute lack of ADA enzymatic activity (12).

DNA purification from the lymphoblastoid cell lines. DNA was harvested by the technique of Chirgwin et al. (15), using guanidine isothiocyanate extraction and purification by cesium chloride gradient centrifugation. DNA was harvested from the interface of the gradients.

Southern DNA analyses. These were done using minor modifications of the standard technique of Southern (16) as described (12).

Genomic cloning. Patient R.P.'s genomic DNA was cut with the Eco RI restriction enzyme and ligated into the EMBL4 lambda DNA arms (Stratagene Cloning Systems, La Jolla, CA). This was packaged using Gigapack Plus (Stratagene) and titered on E. coli P2392. 200,000 plaques were screened by hybridization after transfer to nitrocellulose filters by Benton and Davis plaque hybridization (17). DNA from positive clones was purified by a plate lysate method (18) and recombinants confirmed by restriction endonuclease mapping.

Sequencing. The lambda DNA containing the desired 12-kb Eco RI restriction fragment from patient R.P. was cut with Hind III, and the 4.3-kb Hind III fragment containing the region of the deletion was identified by Southern analysis. This fragment was subcloned into a pUC vector and its identity confirmed by restriction analysis. The 284-bp Sca I-Xba I fragment encompassing the deletion was then 
cloned out of the pUC vector into M13mp19 cut with Sma-Xba I. The M13 clones were transformed into and grown on E. coli JM109. Single-stranded M13 templates were sequenced using a quasi end labeling adaptation of the dideoxy chain termination method (19).

\section{Results}

Northern RNA analyses had previously shown that patient R.P. had no detectable ADA mRNA (Fig. 1 of reference 12). Consistent with this finding, mapping of the patient's ADA gene by Southern DNA analyses demonstrated that he had no detectable sequences hybridizing to an exon 1 genomic probe (reference 12, Fig. 2), although normal restriction fragment
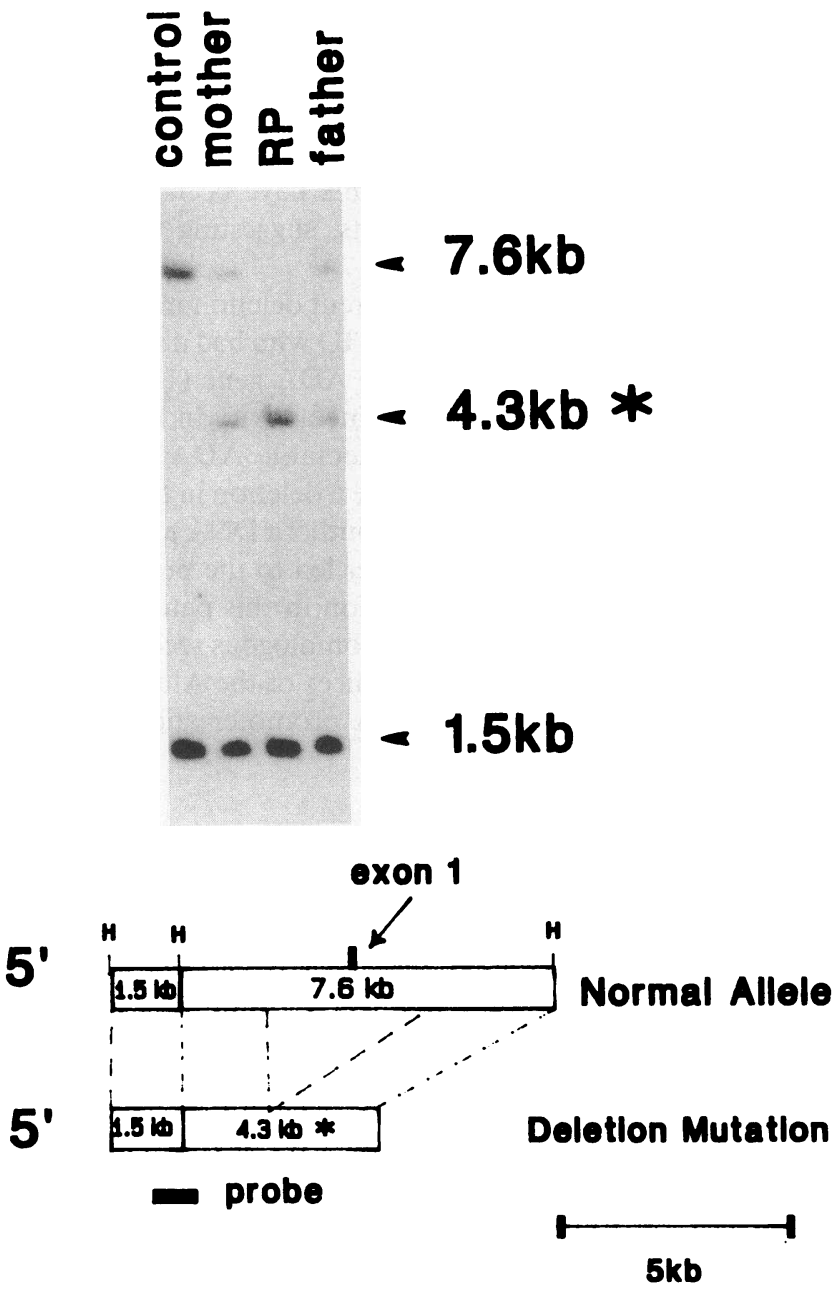

Figure 1. Southern blot of Hind III-digested genomic DNA probed with a fragment cloned from the ADA structural gene. The probe extends from position $-3,935$ to $-3,209$ bp $5^{\prime}$ of the ADA mRNA cap site. Lane 1 contains DNA from a control; lane 2, DNA from R.P.'s mother; lane 3, DNA from R.P.; and lane 4, DNA from R.P.'s father. The diagram at the bottom of the figure shows the 5 ' end of the ADA structural gene. The Hind III restriction sites are indicated as vertical lines and are labeled $H$. Exon 1 is represented as a black box. The 1.5- and 7.6-kb Hind III fragments are located at the $5^{\prime}$ end of the normal ADA gene. The novel 4.3-kb fragment is generated by a $3251 \mathrm{bp}$ deletion of the 7.6-kb fragment. The probe used in this figure spans the Hind III site between the 7.6- and 1.5-kb Hind III fragments. The asterisks indicate the novel 4.3-kb fragment found in patient R.P.

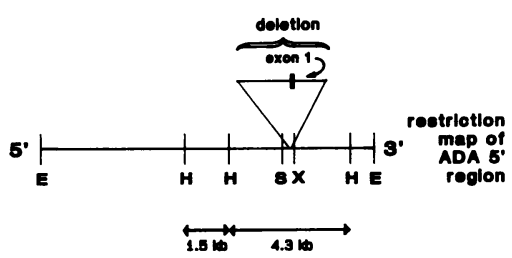

Figure 2. Strategy for cloning R.P.'s DNA. The upper horizontal line represents a $12-\mathrm{kb}$ Eco RI fragment of R.P.'s genomic DNA encompassing the deletion. This restriction fragment extends $5^{\prime}$ $\sim 5.5 \mathrm{~kb}$ from the dele-

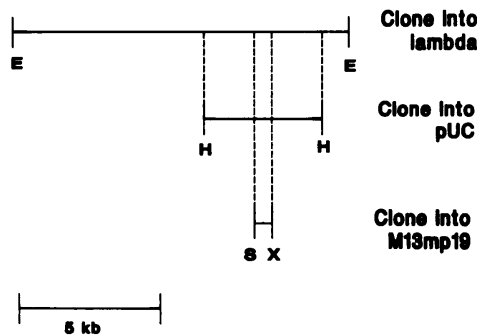
tion site. The 4.3-kb Hind III fragment (see Fig. 1) subcloned into the pUC vector and the 284-bp Sca I-Xba I fragment cloned into M13mp19 are shown. Abbreviations: E, Eco RI; H, Hind III; S, Sca I; X, Xba I.

bands were detected when probed with exons 2-12 in a cDNA. To determine if the patient were homozygous for this mutation, we performed a study of the parents' ADA genes. By using the probe depicted in Fig. 1, we demonstrated that both parents have normal restriction fragments and also a novel fragment compared with controls. The patient has only the novel fragment seen in both parents, suggesting that he is homozygous for a single mutation or has two very similar mutations (see Fig. 1). Most likely, therefore, the parents share a common ancestor.

In further studies (not shown), the deletion joint was mapped between a Sca I site at position $-1,607$ relative to the ADA RNA cap site and an Xba I site in intron 1,2,030 bp 3' to the ADA RNA cap site (numbered according to reference 8). This deletion joint was included within a 12-kb Eco RI restriction fragment in R.P.'s genomic DNA (data not shown).

Fig. 2 shows the strategy used to clone the genomic DNA fragment spanning the deletion junction. Eco RI-digested genomic DNA from patient R.P. was cloned into lambda EMBL4. The 4.3-kb Hind III fragments from two clones were subcloned into a pUC vector and their identity verified by restriction analysis. To prepare DNA for sequencing, the 284bp Sca I-Xba I fragment encompassing the deletion site was cloned into M13mp19 that had been digested with Sma I and Xba I.

Sequencing studies showed that the region $5^{\prime}$ to the recombination joint was derived from the left half of an Alu repeat located 1,491 bp 5' to the ADA mRNA cap site. The region $3^{\prime}$ of the recombination joint was derived from the left half of a different Alu repeat located in the first intron, 1,757 bp 3' to the ADA mRNA cap site. The location of these two Alu repeats and the region deleted is illustrated in Fig. 3.

Sequence analysis revealed that the abnormal allele contains a novel, complete Alu repeat. This Alu repeat was formed by homologous recombination between the two Alu repeats located at either end of the deletion, as shown in Fig. 4. Examination of the published normal ADA gene sequence revealed a 26-bp stretch of nucleotides in both Alu sequences that were involved in the recombination junction. The site of homologous recombination was within this stretch of $26 \mathrm{bp}$, as only one copy is found in the abnormal allele. Detailed analysis of the recombination joint revealed that the " $B$ " sequence of the 


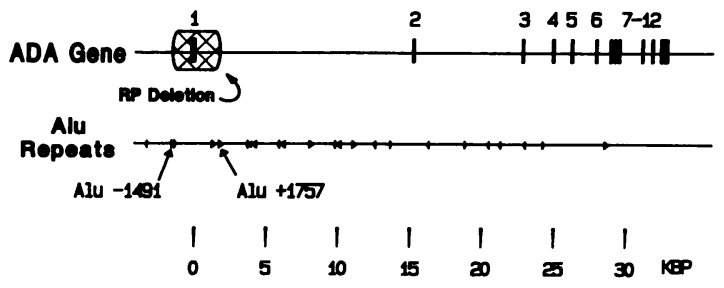

Figure 3. Linear representation of the ADA structural gene. The 12 exons are labeled. The Alu repeats are indicated as carats below the representation of the ADA gene. The area of the deletion is represented by the cross-hatched box. Alu repeats $-1,491$ and $+1,757$ are numbered relative to the ADA mRNA cap site. (This figure is modified from reference 8.)

RNA polymerase III split promoter was included in this region (see Fig. 4). This is the first report of a recombination joint encompassing the " $\mathrm{B}$ " sequence.

\section{Discussion}

This paper defines at the molecular level the first described deletion mutation in a patient with ADA deficiency and SCID. The deletion is 3,248 bp long and encompasses the promoter region and the first exon of the ADA structural gene. Consistent with the critical nature of the region deleted, previous studies have shown that no ADA mRNA is detected in this patient (12). It appears from the Southern analysis in Fig. 2 that the patient is homozygous for this deletion and that both parents are heterozygous. Although the parents are not aware

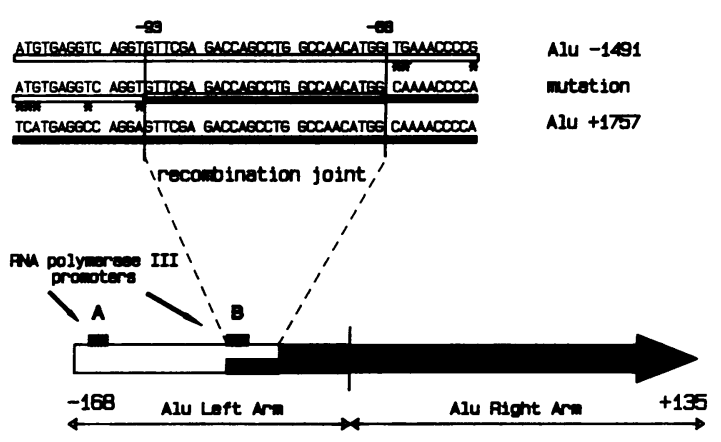

Figure 4. Sequence analysis. The top portion of the figure shows the sequences of the normal Alu at position-1,491 (top sequence, open box), normal Alu at position $+1,757$ (bottom sequence, closed box), and mutant gene (middle sequence) in the region of the recombination joint. (Normal sequences are taken from reference 8 ). The vertical lines at positions -93 and -68 encompass the 26-bp region of the recombination joint. Asterisks between the sequences denote positions at which the basepairs differ. The 26-bp sequence is numbered in accordance with the Alu consensus sequence described by Deininger et al. (22). Lower portion: the patient's novel Alu sequence formed by homologous recombination. Portion contributed by the normal Alu sequence at position $-1,491$ is shown by the open box, and portion contributed by the normal Alu sequence at position $+1,757$ is shown by the closed box. The normal Alu sequence at position $+1,757$ contributes the entire right arm and the $3^{\prime}$ portion of the left arm. The position of the recombination joint in the left half of the Alu consensus repeat is shown. The $5^{\prime} 10 \mathrm{bp}$ of the recombination joint represents the B sequence of RNA polymerase III promoters (27). of common ancestors, they both were born in the same town in Tennessee and likely have a distant ancestor in common.

The ADA promoter region has been sequenced and studied in functional assays in both the human $(8,20)$ and murine $(21)$ systems. The promoter is a typical GC-rich promoter with no TATA or CAAT boxes. Instead, it has multiple putative Spl binding sites $(8,20)$. Various studies suggest that only a few hundred basepairs of promoter sequence are needed $5^{\prime}$ of the ADA mRNA cap site for effective transcription (20-21). The deletion described in this paper, whose $5^{\prime}$ boundary is at position $-1,491( \pm 13 \mathrm{bp})$, encompasses the entire known ADA promoter region and exon 1 and extends to position 1,757 $( \pm 13 \mathrm{bp}$ ) of the first intron (numbered according to reference 8). Thus, the deletion of this important area correlates well with the absolute lack on Northern analysis of any detectable ADA mRNA (12).

Homologous recombination between two Alu repeats was the mechanism of this deletion. Alu repeats have been well characterized as sequences of $\sim 300$ bp (22) which are repeated $\sim 400,000$ times in the human genome (23). They are composed of a right and left half (22). The right half is homologous to the left half and has, in addition, a 31-bp insertion (22). The function, if any, for Alu repeats remains unclear at the present time. They are found scattered throughout the genome; 23 are found in the intron regions of the ADA gene (8). The right halves of Alu repeats are more homologous to each other than the left halves. One would expect, therefore, that the right half would be more frequently involved in homologous recombination events than the left half. This, however, has not been the case in the extensively studied LDL receptor and hemoglobin gene (24-25). In naturally occurring mutations of these genes, Alu sequences have been found at the boundaries of deletions and duplications in homologous and nonhomologous recombination (24). As Lehrman et al. (24) have shown in their review of recombination involving Alu repeats, the majority of recombination events have involved the left half of the Alu repeat sequence, not the more homologous right half. In particular, they noted that most recombination joints occurred between the " $A$ " and " $B$ " sequences of the left arm. These sequences are homologous to the two halves of the RNA polymerase III split promoter (26-27). It is not known whether the particular A and B sequences of the Alu repeats that were involved in this recombination event are transcribed by RNA polymerase III in vivo. Certainly, in vitro, many Alu sequences have been shown to be active in RNA polymerase III transcription assays (27). These promoter-like sequences may play a role in recombination in the human genome, since the majority of recombination events involving Alu repeats have been shown to occur between the A and B sequences. The deletion reported in this paper adds to the group and is the first in which the recombination joint encompasses the $B$ sequence.

One can try to correlate a patient's molecular defect in the ADA structural gene with immunologic phenotype and response to therapy. ADA-deficient patient R.P. is the first ADA-deficient SCID patient whom we have been able to immunologically reconstitute by haploidentical (maternal) stem cell transplantation without pretransplant immunosuppression (reference 2, patient 13; reference 14). This patient's younger brother, who also was born with ADA-deficient SCID, has also achieved normal immune function after a similar haploidentical (maternal) bone marrow stem cell trans- 
plant. Both infants were disparate for a complete HLA haplotype compared with the marrow donor. Thus, the success of these bone marrow transplants cannot be explained by homozygosity at the major histocompatibility locus. One can speculate that an absolute lack of the ADA enzyme might be a favorable predictor for response to bone marrow transplantation. A structural gene deletion mutation as was found in our patient results in an absolute lack of enzyme. This contrasts with the situation found in many patients with point mutations, in whom a minimal amount of enzyme activity may be detected (14). Although this is only speculation, it will be interesting to study additional cases to determine whether patients with gross structural alterations of the ADA gene will also respond to haploidentical bone marrow transplantation.

The R.P. cell line may be useful for studies of ADA gene regulation and ADA gene insertion studies. Introduction of ADA promoter constructs into this cell line should not be complicated by competition from endogenous ADA promoter sequences. Data analysis is also simplified by the lack of endogenous ADA activity and ADA mRNA.

Summary. A deletion mutation encompassing the first exon and promoter region is responsible for ADA deficiency in an infant with SCID. This patient was initially shown to have no ADA enzymatic activity and no ADA mRNA (12). Subsequent Southern analyses have shown that patient R.P. is homozygous for a 3.3-kb deletion encompassing exon 1 and the promoter region. Both parents are heterozygous for the same 3.3-kb deletion. Cloning and sequencing of the recombination joint have shown that the crossover occurred in the left halves of two Alu repeats, one on either side of exon 1. An identical stretch of $26 \mathrm{bp}$ was found in the normal Alu sequences at either end of the deletion. Most likely, this deletion occurred by homologous recombination between the two 26-bp sequences in the Alu repeats. Several examples of homologous recombination involving two Alu repeats have been published involving the LDL receptor and hemoglobin genes (reviewed in reference 24). This is the first, however, involving the B sequence of the RNA polymerase III promoter.

\section{Acknowledgments}

The continuing support and guidance of Dr. Rebecca Buckley is appreciated.

This work was supported by National Institutes of Health grants AI-23791, HD-19919, and DK-38699, by Clinical Research grant 6-433 from the March of Dimes Birth Defects Foundation, and by a Purdue Frederick Fellowship from the American Academy of Allergy and Immunology. Dr. Kaufman is a Leukemia Society of America Scholar.

\section{References}

1. Bortin, M. M., and A. A. Rimm. 1977. Severe combined immunodeficiency disease. Characterization of the disease and results of transplantation. JAMA (J. Am. Med. Assoc.). 238:591-600.

2. Buckley, R. H., S. E. Schiff, H. A. Sampson, R. I. Schiff, M. L. Markert, A. P. Knutsen, M. S. Hershfield, A. T. Huang, G. H. Mickey, and F. E. Ward. 1986. Development of immunity in human severe primary $\mathrm{T}$ cell deficiency following haploidentical bone marrow stem cell transplantation. J. Immunol. 136:2398-2407.

3. Kredich, N. M., and M. S. Hershfield. 1983. Immunodeficiency diseases caused by adenosine deaminase deficiency and purine nu- cleoside phosphorylase deficiency. In The Metabolic Basis of Inherited Disease. J. B. Stanbury, J. B. Wyngaarden, D. S. Fredrickson, J. L. Goldstein, and M. S. Brown, editors. McGraw-Hill Book Co., New York. 1157-1183.

4. Giblett, E. R., J. E. Anderson, F. Cohen, B. Pollara, and H. J. Meuwissen. 1972. Adenosine deaminase deficiency in two patients with severely impaired cellular immunity. Lancet. ii:1067-1069.

5. Orkin, S. H., P. E. Daddona, D. S. Shewach, A. F. Markham, G. A. Brun, S. C. Markham, and W. N. Kelley. 1983. Molecular cloning of human adenosine deaminase gene sequences. J. Biol. Chem. 258:12753-12756.

6. Valerio, D., M. G. C. Duyvesteyn, P. M. Khan, A. G. van Kessel, A. de Waard, and A. J. van der Eb. 1983. Isolation of cDNA clones for human adenosine deaminase. Gene (Amst). 25:231-240.

7. Wiginton, D. A., G. S. Adrian, R. L. Friedman, D. P. Suttle, and J. J. Hutton. 1983. Cloning of cDNA sequences of human adenosine deaminase. Proc. Natl. Acad. Sci. USA. 80:7481-7485.

8. Wiginton, D. A., D. J. Kaplan, J. C. States, A. C. Akeson, C. M. Perme, I. J. Bilyk, A. J. Vaughn, D. L. Lattier, and J. J. Hutton. 1986. Complete sequence and structure of the gene for human adenosine deaminase. Biochemistry. 25:8234-8244.

9. Adrian, G. S., D. A. Wiginton, and J. J. Hutton. 1984. Structure of adenosine deaminase mRNAs from normal and adenosine deaminase-deficient human cell lines. Mol. Cell. Biol. 4:1712-1717.

10. Bonthron, D. T., A. F. Markham, D. Ginsberg, and S. H. Orkin. 1985. Identification of a point mutation in the adenosine deaminase gene responsible for immunodeficiency. J. Clin. Invest. 76:894897.

11. Valerio, D., B. M. M. Dekker, M. G. C. Duyvesteyn, L. van der Voorn, Th. M. Berkvens, H. van Ormondt, and A. J. van der Eb. 1986. One adenosine deaminase allele in a patient with severe combined immunodeficiency contains a point mutation abolishing enzyme activity. EMBO (Eur. Mol. Biol. Organ.) J. 5:113-119.

12. Markert, M. L., M. S. Hershfield, D. A. Wiginton, J. C. States, F. E. Ward, S. H. Bigner, R. H. Buckley, R. E. Kaufman, and J. J. Hutton. 1987. Identification of a deletion in the adenosine deaminase gene in a child with severe combined immunodeficiency. J. Immunol. 138:3203-3206.

13. Katsuki, T., and Y. Hinuma. 1975. Characteristics of cell lines derived from human leukocytes transformed by different strains of Epstein-Barr virus. Int. J. Cancer. 12:203-210.

14. Markert, M. L., M. S. Hershfield, R. I. Schiff, and R. H. Buckley. 1987. Adenosine deaminase and purine nucleoside phosphorylase deficiencies: evaluation of therapeutic interventions in eight patients. J. Clin. Immunol. 7:389-399.

15. Chirgwin, J. M., A. E. Przybyla, R. J. McDonald, and W. J. Rutter. 1979. Isolation of biologically active ribonucleic acid from sources enriched in ribonuclease. Biochemistry. 24:5294-5299.

16. Southern, E. M. 1975. Detection of specific sequences among DNA fragments separated by gel electrophoresis. J. Mol. Biol. 98:503-517.

17. Benton, W. D., and R. W. Davis. 1977. Screening Lambda gt recombinant clones by hybridization of single plaques in situ. Science (Wash. DC). 196:180-182.

18. Maniatis, T., E. F. Fritsch, and J. Sambrook. 1982. Molecular Cloning: A Laboratory Manual. Cold Spring Harbor Laboratory, Cold Spring Harbor, NY. 371-372.

19. Brunner, A. M., J. C. Schimenti, and C. H. Duncan. 1986. Dual evolutionary modes in the bovine blobin locus. Biochemistry. 25:5028-5035.

20. Valerio, D., M. G. C. Duyvesteyn, B. M. M. Dekker, G. Weeda, Th. M. Berkvens, L. van der Voorn, H. van Ormondt, and A. J. van der Eb. 1985. Adenosine deaminase: characterization and expression of a gene with a remarkable promoter. EMBO (Eur. Mol. Biol. Organ.) J. 4:437-443. 
21. Ingolia, D. E., M. R. Al-Ubaidi, C.-Y. Yeung, H. A. Bigo, D. A. Wright, and R. E. Kellems. 1986. Molecular cloning of the murine adenosine deaminase gene from a genetically enriched source: identification and characterization of the promoter region. Mol. Cell. Biol. 6:4458-4466.

22. Deininger, P. L., D. J. Jolly, C. M. Rubin, T. Friedmann, and C. W. Schmid. 1981. Base sequence studies of 300 nucleotide renatured repeated human DNA clones. J. Mol. Biol. 151:17-33.

23. Rinehart, F. P., T. G. Ritch, P. L. Deininger, and C. W. Schmid. 1981. Renaturation rate studies of a single family of interspersed repeated sequences in human deoxyribonucleic acid. Biochemistry. 20:3003-3010.

24. Lehrman, M. A., D. W. Russell, J. L. Goldstein, and M. S.
Brown. 1987. Alu-Alu recombination deletes splice acceptor sites and produces secreted low density lipoprotein receptor in a subject with familial hypercholesterolemia. J. Biol. Chem. 262:3354-3361.

25. Nicholls, R. D., N. Fischel-Ghodsian, and D. R. Higgs. 1987. Recombination at the human alpha-globin gene cluster: sequence features and topological constraints. Cell. 49:369-378.

26. Galli, G., H. Hofstetter, and M. L. Birnstiel. 1981. Two conserved sequence blocks within eukaryotic tRNA genes are major promoter elements. Nature (Lond.). 294:626-631.

27. Paolella, G., M. A. Lucero, M. H. Murphy, and F. E. Baralle. 1983. The Alu family repeat promoter has a tRNA-like bipartite structure. EMBO (Eur. Mol. Biol. Organ.) J. 2:691-696. 